\title{
Optical Phase Conjugation in a Silicon Waveguide with Lateral p-i-n Diode for Nonlinearity Compensation
}

Da Ros, Francesco; Gajda, Andrzej; Porto da Silva, Edson; Peczek, Anna; Mai, Andreas; Petermann, Klaus; Lars, Zimmermann; Oxenløwe, Leif Katsuo; Galili, Michael

Published in:

Journal of Lightwave Technology

Link to article, DOI:

10.1109/JLT.2018.2873684

Publication date:

2018

Document Version

Peer reviewed version

Link back to DTU Orbit

Citation $(A P A)$ :

Da Ros, F., Gajda, A., Porto da Silva, E., Peczek, A., Mai, A., Petermann, K., Lars, Z., Oxenløwe, L. K., \& Galili, M. (2018). Optical Phase Conjugation in a Silicon Waveguide with Lateral p-i-n Diode for Nonlinearity

Compensation. Journal of Lightwave Technology, 37(2), 323 - 329 . https://doi.org/10.1109/JLT.2018.2873684

\section{General rights}

Copyright and moral rights for the publications made accessible in the public portal are retained by the authors and/or other copyright owners and it is a condition of accessing publications that users recognise and abide by the legal requirements associated with these rights.

- Users may download and print one copy of any publication from the public portal for the purpose of private study or research.

- You may not further distribute the material or use it for any profit-making activity or commercial gain

- You may freely distribute the URL identifying the publication in the public portal 


\title{
Optical Phase Conjugation in a Silicon Waveguide with Lateral p-i-n Diode for Nonlinearity Compensation
}

\author{
F. Da Ros, Member, IEEE, OSA, A. Gajda, E.P. da Silva, Member, IEEE, A. Pęczek, \\ A. Mai, K. Petermann, Life Fellow, IEEE, Member, OSA, L. Zimmermann, \\ L.K. Oxenløwe, Member, OSA, and M. Galili, Member, IEEE
}

\begin{abstract}
In-line optical phase conjugation is a well-known technique to enhance the received signal quality through nonlinearity compensation. Being able to implement the conjugation in cm-scale highly nonlinear devices, which can be integrated on a silicon chip could potentially lead to several benefits in terms of small footprint and co-integration with linear signal processing functionalities, as well as lower power consumption. Here, we focus on silicon waveguides to implement the optical phase conjugation through four-wave mixing. The challenges in terms of conversion efficiency imposed by the presence of nonlinear loss are tackled by using a lateral $\mathrm{p}-\mathrm{i}-\mathrm{n}$ diode along the waveguide. When the diode is reverse biased, the conversion efficiency can be effectively enhanced by the decrease in free-carrier absorption. Low-penalty conversion can therefore be achieved for WDM signals and the highquality of the generated idlers is critical in demonstrating a 1-dB Q-factor improvement through optical phase conjugation in a 5 WDM channel 16-QAM transmission system after 644$\mathrm{km}$ of dispersion compensated transmission. The performance improvement enables a performance better than the HD-FEC threshold for all the transmitted channels.
\end{abstract}

Index Terms - four-wave mixing, integrated waveguides, quadrature amplitude modulation, coherent communications.

\section{Introduction}

$\mathrm{S}$ EVERAL nonlinearity compensation techniques have been extensively investigated in the recent years to tackle the challenge of Kerr nonlinearity and the strong limitation it poses towards increasing the spectral efficiency and data rates of current communication systems [1, [2]. The challenge arises from the need to maintain a high optical signal-to-noise ratio (OSNR) throughout the transmission, as required by more advanced signaling schemes, leading to a higher launched power

F. Da Ros, L.K. Oxenløwe and M. Galili are with the Department of Photonics Engineering, Technical University of Denmark, Kongens Lyngby, 2800 Denmark, e-mail: fdro@fotonik.dtu.dk.

A. Gajda and A. Pęczek are with IHP Solutions GmbH, Im Technologiepark 25, 15236 Frankfurt (Oder), Germany, email: andrzej.gajda@ihp-solutions.com.

E.P. da Silva was with the Department of Photonics Engineering, Technical University of Denmark, Kongens Lyngby, 2800 Denmark. $\mathrm{He}$ is now with the Department of Electrical Engineering of the Federal University of Campina Grande (UFCG), Paraba, Brazil, email:edson.silva@dee.ufcg.edu.br.

A. Mai and L. Zimmermann are with IHP, Im Technologiepark 25, 15236 Frankfurt (Oder), Germany.

K. Petermann and L. Zimmermann are with the Institut für Hochfrequenz- und Halbleiter-Systemtechnologien, TU Berlin, Einsteinufer 25, Berlin Germany. being required and causing the onset of Kerr-induced nonlinear distortion. Digital $[3]-8]$ and all-optical 6 , [9] -17] approaches, as well as preliminary work where techniques from both fields are combined [18], have been reported. One key advantage of all-optical approaches is the operation bandwidth, which enables to process several WDM channels simultaneously. Additionally, the access to a broad bandwidth allows for compensation of the intrachannel nonlinear interaction, which would be challenging to address at the receiver side when only one channel is processed. Using optical phase conjugation (OPC), nonlinear compensation over a record bandwidth up to $2.3 \mathrm{THz}$ has been reported [16], which is well beyond the capabilities of practical coherent receivers. The majority of OPC demonstrations so far have relied on implementing the conjugation through four-wave mixing (FWM) in a highly nonlinear fiber (HNLF) [6], 9$]-[15]$ or cascaded second-order effects in periodically-poled lithium niobate (PPLN) 16.

The choice of fiber and PPLN as material platforms has been mainly dictated by the good level of maturity reached by the respective fabrication technologies. However, silicon photonics has experienced a significant growth over the past few years following a push towards increased integration, especially in terms of photonics and electronics, as well as the potential to leverage from mature fabrication techniques developed within the CMOS technology 19-21]. From a nonlinear signal processing perspective, silicon has the considerable benefits of a high nonlinear refractive index and strong light confinement which further enhances the nonlinear interaction. The stronger nonlinearity requires only $\mathrm{cm}$-scale devices, similarly to PPLN, and thus several order of magnitude shorter than HNLFs where usually hundreds of meter of length are necessary. However, unlike PPLN, silicon has a strong potential for on-chip monolithic integration, thus combining linear and nonlinear processing, as well as optics and electronics, on the same chip. Additionally, as group velocity dispersion is dominated by the waveguide dispersion, by tuning the waveguide cross-section, the dispersion, and thus the conversion bandwidth, can be optimized. Nevertheless, whereas using silicon for linear operations has been extremely successful 19]-[21, a number of challenges has limited the appreciation of silicon for 
nonlinear signal processing. The material bandgap results in a strong two-photon absorption (TPA) at telecom wavelengths $(1550 \mathrm{~nm})$. Other than introducing additional loss, TPA leads to the accumulation of free carriers within the core region of the waveguide. This excess of free carriers results in even higher loss through free-carrier absorption (FCA). The combined impact of TPA and FCA effectively limits the achievable efficiency of four-wave mixing (FWM) in silicon waveguides. This was in fact the key limitation preventing performance improvement in a recent first attempt at providing nonlinearity compensation through OPC in a silicon waveguide [17. The successful compensation of nonlinear distortion was indeed shown, but the poor conversion efficiency (CE) and thus limited achievable optical signal-to-noise ratio (OSNR) of the conjugate signal prevented a Q-factor improvement at optimum launched power, when compared with straight transmission. Whereas reducing TPA in silicon would require changingthe material properties, for example by looking at amorphous structures $22-24$ or focusing on silicon-based compound materials $[24]-27$, the impact of FCA can be significantly reduced simply by adding $\mathrm{n}$ - and p-doped regions across the waveguide. When a reverse bias voltage is applied to these doped regions, the generated electric field sweeps out the accumulated free carriers reducing the nonlinear loss due to FCA $[28-30$.

In this work, we adopt this approach by focusing on silicon nano-rib waveguides with a lateral p-i-n diode structure. The diode structure enables the effective suppression of FCA [28]-30], thus yielding higher CE and idler OSNR. In particular, we extend our recent demonstration in 15 by providing a thorough characterization of the nonlinear medium and an extensive discussion of the system demonstration that has lead to a 1-dB Q-factor improvement through nonlinearity compensation for a 5-channel wavelength division multiplexed (WDM) 16-quadrature amplitude modulated (QAM) single-polarization system after $644-\mathrm{km}$ of dispersion compensated transmission.

The paper is structured as follows. In Section III, fabrication and static FWM characterization of the silicon waveguide is discussed underlining the optimization in terms of waveguide length, pump power and reverse bias voltage that enabled to achieve sufficient $\mathrm{CE}$ for the following system demonstration. In Section III the experimental setup for the OPC stage based on the silicon waveguide and the system setup to evaluate its performance are presented, followed by Section [V] where the performance is reported and discussed by benchmarking the converted idler against the original signal. In Section $\mathrm{V}$ the transmission system used for the experimental demonstration is presented and the results of the comparison between straight and midlink OPC transmission are discussed. Finally, Section VI summarizes the key results.

\section{Waveguide fabrication and characterization}

The silicon waveguide used in this work has been fabricated in the BiCMOS pilot line of IHP GmbH. The waveguides are manufactured on a silicon-on-insulator

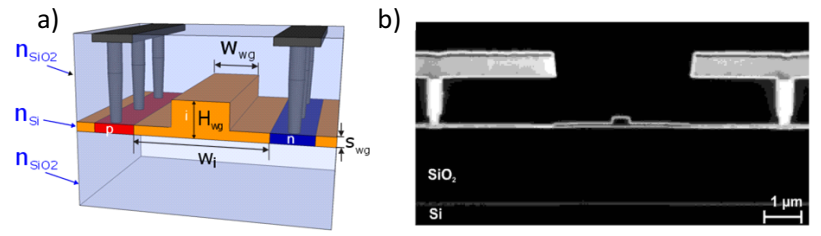

Fig. 1. (a) Waveguide design and (b) SEM image of the fabricated nano-rib waveguide showing the vertical electrical contact to the doped region of the p-i-n junction.

(SOI) wafer with a $220-\mathrm{nm}$ top silicon layer and a $2 \mu \mathrm{m}$ buffer oxide. A lithographic step with deep UV lithography patterns the silicon layer, which is subsequently etched to provide a nano-rib waveguide with a width of 500 $\mathrm{nm}$ and a slab thickness of $100 \mathrm{~nm}$. Note that these cross-section dimensions lead to normal dispersion in the telecommunication C-band, thus limiting the achievable FWM bandwidth (as discussed in the following). Preliminary attempts to improve the design and achieve anomalous dispersion have already lead to promising results in terms of broader bandwidth (up to $40 \mathrm{~nm}$ for a cross-section of $500 \mathrm{~nm} \times 400 \mathrm{~nm}$ ), however at the expense of higher propagation loss and thus lower input-to-output conversion efficiency 31. Since the main focus of this work is the demonstration of nonlinearity compensation through OPC in a transmission scenario, bandwidth has been sacrificed in favor of output idler power and the crosssection leading to normal dispersion has been chosen. The structure of the waveguide as well as an SEM image of the fabricated device are shown in Fig. 1. The separation between $\mathrm{p}$ - and $\mathrm{n}$ - doped regions has been optimized to $1.2 \mu \mathrm{m}[29]$.

In order to interface the waveguides with optical fibers, vertical 1D grating couplers have been used. The simple design of these couplers makes them strongly polarizationsensitive, thus limiting the further discussion to singlepolarization signals. This limitation can be overcome by using a coupling scheme that can accept both TE and TM polarizations 32 or by using a polarization-diversity scheme 33. The insertion loss of the grating coupler is approx. $4.8 \mathrm{~dB}$ /coupler and the linear propagation loss is optimized to less than $1 \mathrm{~dB} / \mathrm{cm}$ when a $40-\mathrm{V}$ reverse-bias voltage is applied to the p-i-n diode. As discussed in 30, the diode helps also in reducing the linear propagation loss by decreasing the carrier life-time for surface states.

Several waveguide lengths have been considered, spanning from $2 \mathrm{~cm}$ to $5 \mathrm{~cm}$, in order to investigate the optimum waveguide lengths for implementing the optical phase conjugation stage. In order to characterize the quality of the conversion, a strong continuous-wave (CW) pump was coupled into the waveguide together with a weak $\mathrm{CW}$ and the optical spectra at the output of the waveguide were recorded to extract the power of the newly generated idler. For all the analysis, given the polarizationsensitivity of FWM and of the waveguide grating coupler, the polarization of the two optical waves was manually matched to the TE mode of the waveguide. 

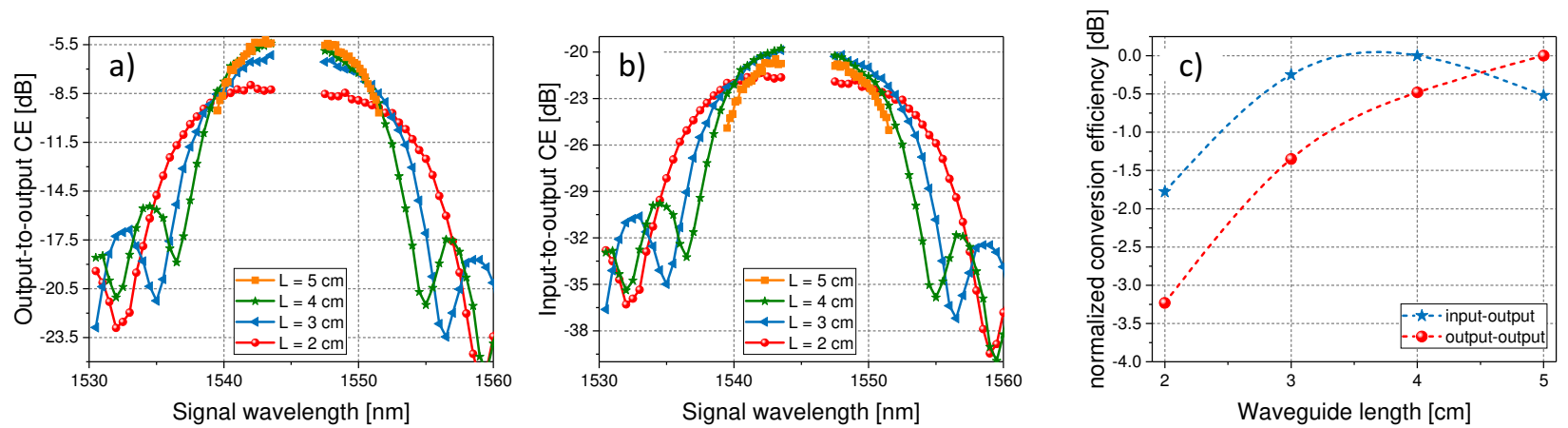

Fig. 2. Optimization of the waveguide length: output-to-output (a) and input-to-output conversion efficiency spectra for different waveguide lengths at an input pump power (at the grating coupler) of $26 \mathrm{dBm}$. (c) Normalized conversion-efficiency as a function of the waveguide length for a 1543-nm signal showing the optimum when considering the input-to-output efficiency.

The results of the waveguide length optimization are shown in Fig. 2 . In Fig. 2(a), the output-to-output CE, defined as the power ratio between idler and signal at the waveguide output, is shown as a function of the signal wavelength for the waveguide lengths considered. For all the curves, pump power, pump wavelength and reverse bias voltage have been kept at $26 \mathrm{dBm}, 1545.5 \mathrm{~nm}$ and $-40 \mathrm{~V}$, respectively. The measured curves show the expected increase in $\mathrm{CE}$ as the waveguide length increases. Similarly, the accumulated dispersion increases yielding a narrowing of the conversion bandwidth for longer waveguides. Whereas this result seems to indicate that longer waveguides are desirable, considering the input-to-output $\mathrm{CE}$, i.e. the power ratio between output idler and input signal, a trade-off between FWM efficiency and insertion loss is evident. This trade-off is shown in Fig. 2(b) for the full CE spectra and then clarified in Fig. 2(c) for a single signal wavelength $(1543 \mathrm{~nm})$ chosen close to the pump, not to be affected by the conversion bandwidth narrowing with the length. The two figures, clearly show $4 \mathrm{~cm}$ to be the best available waveguide length. Longer waveguides provide slightly higher $\mathrm{CE}$ but at the expense of higher IL, which leads to an overall decrease of the output idler power.

In the following characterization and system demonstration, a waveguide length of $4 \mathrm{~cm}$ was chosen. The total (fiber-to-fiber) linear insertion loss of the waveguide, measured at low-power to avoid the impact of TPA and FCA were $13.4 \mathrm{~dB}$. This led to an available conversion bandwidth of $10 \mathrm{~nm}$, which, while sufficient for this demonstration, would require further dispersion engineering for practical applications in the context of a fully-loaded transmission system 31 .

The other parameters of the OPC stage that were optimized are the reverse bias voltage and the pump power. The input-to-output $\mathrm{CE}$ at a fixed pump power (26 $\mathrm{dBm}$ at the input grating coupler) is shown in Fig. 3(a) for several reverse bias voltages. As can be seen, by increasing the bias voltage, the FWM efficiency is significantly improved until it saturates at approx. -30 to $-40 \mathrm{~V}$.

The input-to-output $\mathrm{CE}$ at $-40-\mathrm{V}$ of reverse bias is shown in Fig 3(b). The CE increases more than linearly with the input pump power $(\approx 4 \mathrm{~dB}$ increase for a $3 \mathrm{~dB}$ pump power increases), approaching the theoretical 6 - $\mathrm{dB}$ increase in the absence of all nonlinear loss and pump depletion. This further confirms the effectiveness of the diode in reducing the nonlinear loss. The available pump power was limited by our experimental setup and not by nonlinear loss nor by the power handling of the device. By using higher pump power, higher $\mathrm{CE}$ values have already been shown 30.

\section{Optical phase conjugation stage}

The full experimental setup of the OPC stage is shown in Fig. 4, together with the 5-channel WDM transmitter and receiver used to evaluate the performance of the converted idler and benchmark it against the original signal.

The transmitter is based on five WDM channels on a 25$\mathrm{GHz}$ grid. The central channel and the four neighboring interferers are generated by encoding 16-GBd 16-QAM signals onto the optical carriers from external cavity lasers (ECLs, 10-kHz linewidth) by using standard IQ modulators (one for the central channel and one for the four neighbors). The modulators are driven by an arbitrary waveform generator (AWG) loaded with random data extracted from a pseudo-random bit sequence (PRBS) and pulse shaped with root-raised cosine with a roll-off factor of 0.2. After combining the five channels and amplifying them in an erbium-doped fiber amplifier (EDFA), the channels are further individually decorrelated by using a decorrelation stage based on wavelength selective switches (WSSs) and fiber-based delays for a minimum interchannel decorrelation of more than 70 symbols. The decorrelation stage further enables the separate tuning of the state-of-polarization of each channel in order to ensure that they are all polarization aligned at the output of the transmitter. The output of the transmitter is then injected into the OPC stage, where the signals are further amplified and out-of-band noise is removed with optical bandpass filters (OBPFs). The pump, from another ECL, is also amplified and band-pass filtered (0.8-nm wide OBPF) before being combined with the signals and injected into 

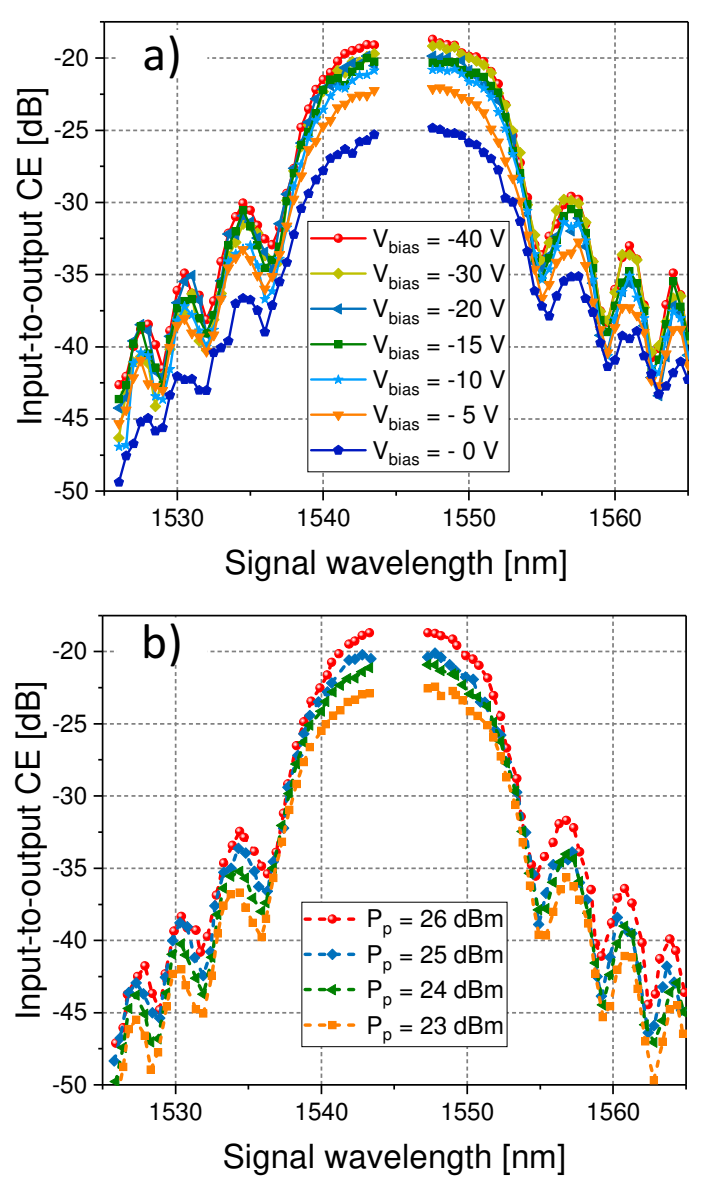

Fig. 3. Input-to-output conversion efficiency spectra (a) for different reverse bias voltages at a fixed input pump power of $26 \mathrm{dBm}$ at the grating coupler and (b) for different input pump powers at a fixed reverse bias voltage of $-40 \mathrm{~V}$.

the waveguide through the vertical grating couplers. In order to maximize the output idler power (i.e. the CE), the polarization of signals and pump are aligned to the TE mode of the waveguide. At the waveguide output, a pair of OBPFs interspersed with an EDFA are used to suppress the original signals and pump and select the idler wavelengths. An optical spectrum at the output of the waveguide, prior to filtering, is depicted in the inset of Fig. 4 showing an output-to-output $\mathrm{CE}$ of $-6 \mathrm{~dB}$ (inputto-output $\mathrm{CE}$ of $-19.5 \mathrm{~dB}$ ). The slightly lower number is due to the lower pump power of $25 \mathrm{dBm}$ used for the system demonstration as it was still sufficient to ensure low OSNR degradation in the conversion.

At the receiver side, a standard pre-amplified singlepolarization coherent receiver is used, consisting mainly of a $10-\mathrm{kHz}$ linewidth local oscillator (LO) and an $80-\mathrm{GSa} / \mathrm{s}$ sampling oscilloscope providing the analog-to-digital conversion. After conversion, standard off-line digital signal processing (DSP) consisting of low-pass filtering, radiusdirected equalization with a multi-modulus algorithm (MMA), carrier recovery with a digital phase-locked loop (DPLL) and bit error ratio (BER) counting, is applied. In order to provide statistically-relevant measurements, more than $8 \times 10^{5}$ symbols were processed, leading to a reliable BER above $10^{-5}$ (Q-factor $\approx 12.6 \mathrm{~dB}$ ).

\section{Converter performance}

First of all, the input power to the OPC stage has been optimized according to the trade-off between loss of OSNR in the conversion (due to the limited $\mathrm{CE}$ and pump noise leakage) and the on-set of nonlinear effects such as cross and self phase modulation (XPM, SPM) on the signal. The results of the optimization, carried out by varying the input signal power into the OPC stage and measuring the corresponding Q-factor extracted from the measured BER, are shown in Fig. 5(a). Whereas the total idler OSNR ratio increases linearly with the input signal power, the performance peaks at an input signal power of approx. $13.5 \mathrm{dBm}(6.5 \mathrm{dBm} /$ channel $)$. This input power level corresponds to a received OSNR of approx. $34 \mathrm{~dB}$. By keeping the signal power into the converter set to $13.5 \mathrm{~dB}$, the performance of the idler was benchmarked against the signal at the output of the transmitter by varying the received OSNR through noise loading with amplified spontaneous emission (ASE) noise at the receiver input. The BER as a function of the receiver OSNR is shown in Fig. 5(b). The curves shown refer to the central channel with the neighbors having very similar performance. The penalty from the conversion is below $0.5 \mathrm{~dB}$ throughout most of the OSNR range considered and specifically at the hard-decision forward error correction (HD-FEC) threshold of BER $=3.8 \times 10^{-3}$ ( $7 \%$ overhead). Notice that in the specific analysis, no additional noise loading is performed at the input of the OPC stage. This can be considered a potential worst case scenario in terms of signal degradation. As the conversion will occur in the middle of the transmission link, the signal will have suffered OSNR degradation throughout the transmission and therefore the penalty is expected to be lower than the $0.5 \mathrm{~dB}$ measured with a clean signal at the transmitter output 34 .

\section{OPC-based transmission}

The full transmission testbed used to evaluate the improvement provided by the OPC stage based on the $\mathrm{Si}$ waveguide is shown in Fig. 6(a) with the transmitter and receiver already shown in Fig. 4. At the output of the transmitter, the signals are injected into a recirculating transmission loop based on three fully dispersioncompensated spans and EDFAs to compensate for the fiber loss. Each span consists of a first spool of standard single mode fiber (SMF) followed by a spool of dispersion compensating fiber (DCF) such that the accumulated dispersion per span is fully compensated. The linear loss, nonlinear coefficient and dispersion coefficient was $0.2 \mathrm{~dB} / \mathrm{km}, 1.2 / \mathrm{W} / \mathrm{km}$ and $16.5 \mathrm{ps} / \mathrm{nm} \cdot \mathrm{km}$ for the SMF, $0.58 \mathrm{~dB} / \mathrm{km}, 7.6 / \mathrm{W} / \mathrm{km}$ and $-120 \mathrm{ps} / \mathrm{nm} \cdot \mathrm{km}$ for the DCF. The three spans have slightly different cummulative lengths of $50 \mathrm{~km}, 61 \mathrm{~km}$ and $50 \mathrm{~km}$, respectively. At each loop turn, acusto-optical modulators (AOMs, acting 


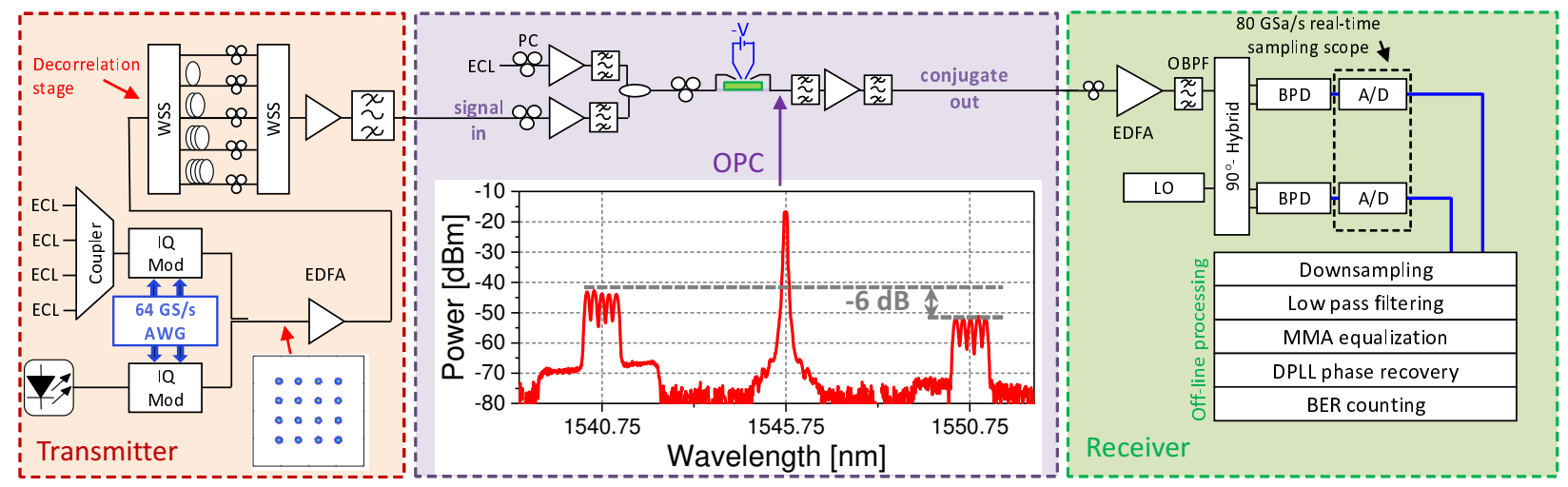

Fig. 4. Experimental setup for benchmarking the performance of the wavelength converted idler against the original (back-to-back) signal. Insets show the constellation diagram at the transmitter and the optical spectra at the output of the waveguide for a pump power of $25 \mathrm{dBm}$.
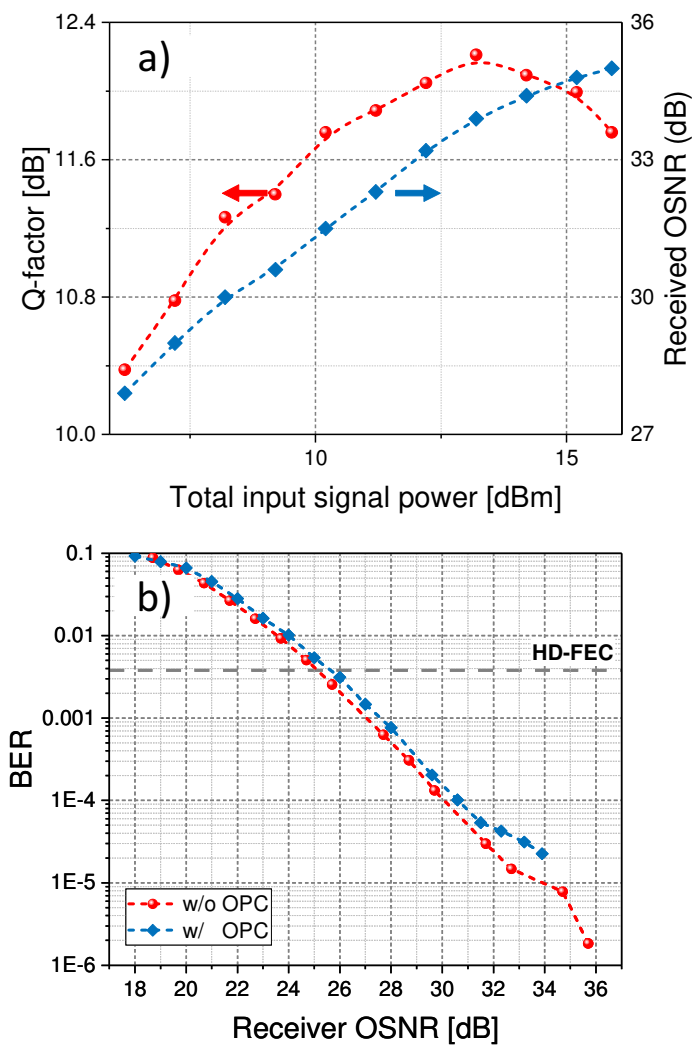

Fig. 5. Optical phase conjugation performance: (a) Q-factor of the central channel and received OSNR as a function of the input signal power and (b) BER versus receiver OSNR for back-to-back signal (w/o OPC) and converted idler (w/ OPC) at the optimum input signal power of $13.5 \mathrm{dBm}$.

as shutters) enable to connect either the OPC path or a straight parallel path consisting of an additional EDFA compensating for the loss of the additional loop components and a gain flattening filter (GFF) which also suppresses out-of-band ASE noise. When going through the OPC path, the loss of the additional loop components are compensated for by the EDFA at the waveguide output (Fig. 4) while the input EDFA is set such that the optimum signal power is injected into the waveguide.
A total of four recirculations were considered, corresponding to $644 \mathrm{~km}$, before the WDM channels were sent to the single-polarization receiver. In the case of OPCbased transmission, the OPC stage was placed in the middle of the link, i.e. after $322 \mathrm{~km}$. In order to achieve so, the OPC AOM (AOM \#3 in Fig. 6) (a) let light through only after the second recirculation, whereas during the other three recirculations (1,3 and 4) the signal band (or idler band in the case of third and forth recirculation) passed through the upper branch with the EDFA and GFF. A scheme of the equivalent in-line setup is shown in Fig. 6(b).

Remark that the use of a recirculating loop for transmission of single polarization signals generally require extra care in considering the polarization evolution within the loop 35] In this work, the polarization controllers at the transmitter side (Fig. 4) allowed to both polarizationalign the signal and to optimize the state-of-polarization (SOP) in the loop such that it was stable over the propagation 35. The state-of-polarization of each channel was further monitored at the OPC stage where any polarization misalignment would result in a lower $\mathrm{CE}$ for the specific channel. The $\mathrm{CE}$ was therefore equalized by rotating the overall SOP of the full channel band without affecting the co-polarization of the channels. Additionally, the impact of polarization-mode dispersion (PMD) was negligible due to the relatively short transmission distance and narrow signal band. For more broad-band, longer reach systems, PMD is a well-known challenge for OPCbased nonlinearity compensation. As discussed in [36, multiple OPC spaced less than half of the polarization correlation length can address the problem, while providing additional gain from the multiple conjugations.

The improvement provided by OPC through nonlinearity compensation is evaluated by comparing the performance of the straight transmission (no OPC) with the mid-link OPC scenario. The Q-factor of the central channel is reported in Fig. 7(a) for both transmission conditions as a function of the launched power per channel at the input of each span. In the linear transmission 

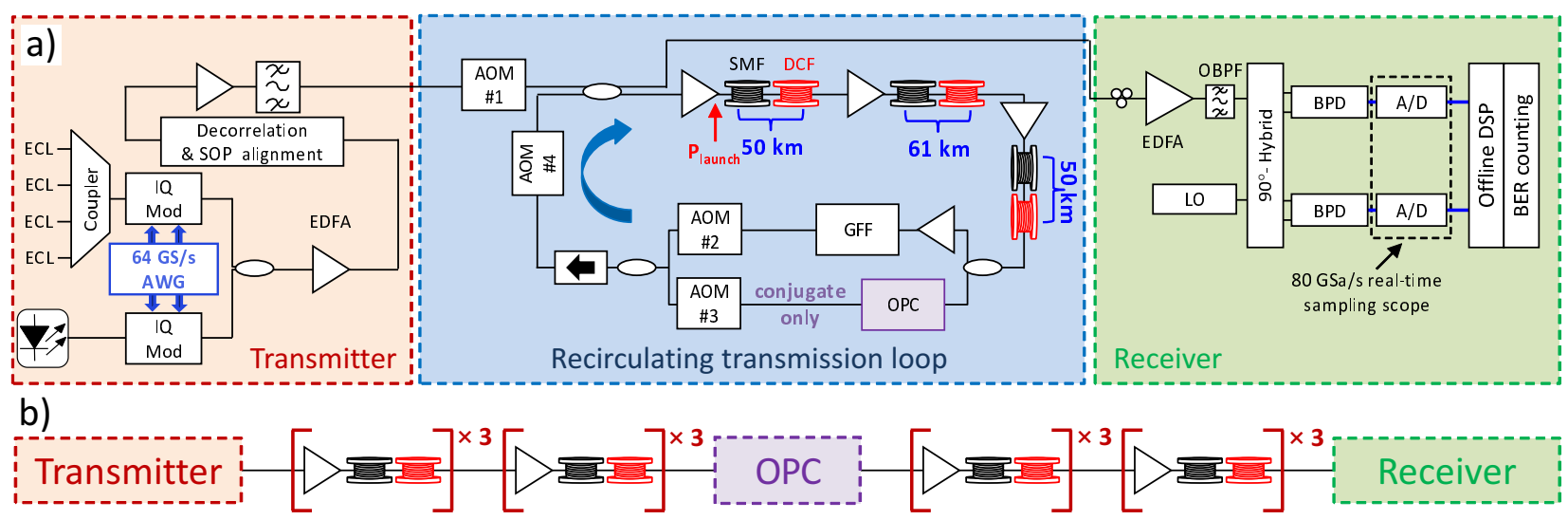

Fig. 6. (a) Experimental transmission setup to evaluate the improvement provided by mid-link OPC in terms of nonlinearity compensation compared to straight (no OPC) transmission; (b) functionally equivalent in-line transmission setup.

regime, no appreciable difference can be seen between straight and mid-link OPC, further confirming the low penalty introduced by the conversion, consistent with Fig. 5(b). As the launched power is increased, however, the impact of nonlinearity starts degrading the signal performance leading to an optimum launch power of approx. $-8 \mathrm{dBm} /$ channel and $-6 \mathrm{dBm} /$ channel for straight and OPC-based transmission, respectively. The increase of optimum launched power when the OPC is introduced confirms the effective nonlinearity compensation which results from the conversion. Additionally, the presence of the OPC yields a 1-dB improvement in Q-factor for the central channel. The signal quality enhancement provided by the OPC over straight transmission can be clearly seen in the constellation diagrams in inset of Fig. 7(a). Such an improvement is maintained for four of the five channels, as reported in Fig. 7(b) where the maximum Q-factors for each transmission scenario (i.e. at $-8 \mathrm{dBm}$ and $-6 \mathrm{dBm}$ respectively) are shown for all channels. As can be seen, the OPC stage enables achieving Q-factors above the HDFEC $\left(B E R=3.8 \times 10^{-3}\right.$ or Q-factor $\left.=8.53 \mathrm{~dB}\right)$ threshold for all the channels. This performance was not achievable in the straight configuration. All the channels are improved by the OPC, except for the highest frequency channel (194.66 THz), which is slightly degraded. This is due to the residual gain tilt in the EDFAs. In straight transmission, such a channel has the best performance due to higher OSNR. After conversion the band mirroring effect due to FWM, positions the channel at the lowest frequency where noise accumulation is higher. This slight degradation is, however, balanced by the almost $2.5 \mathrm{~dB}$ of improvement for the lowest frequency channel (194.56 THz) which undergoes the opposite shift (lowest to highest frequency), due to the OPC. Therefore, the frequency-mirroring of the signal band performed by the OPC helps counteracting the residual gain tilt of the EDFAs.

\section{Conclusion}

The use of optical phase conjugation is a well-known technique to enhance the received signal quality through nonlinearity compensation. In this work we presented an
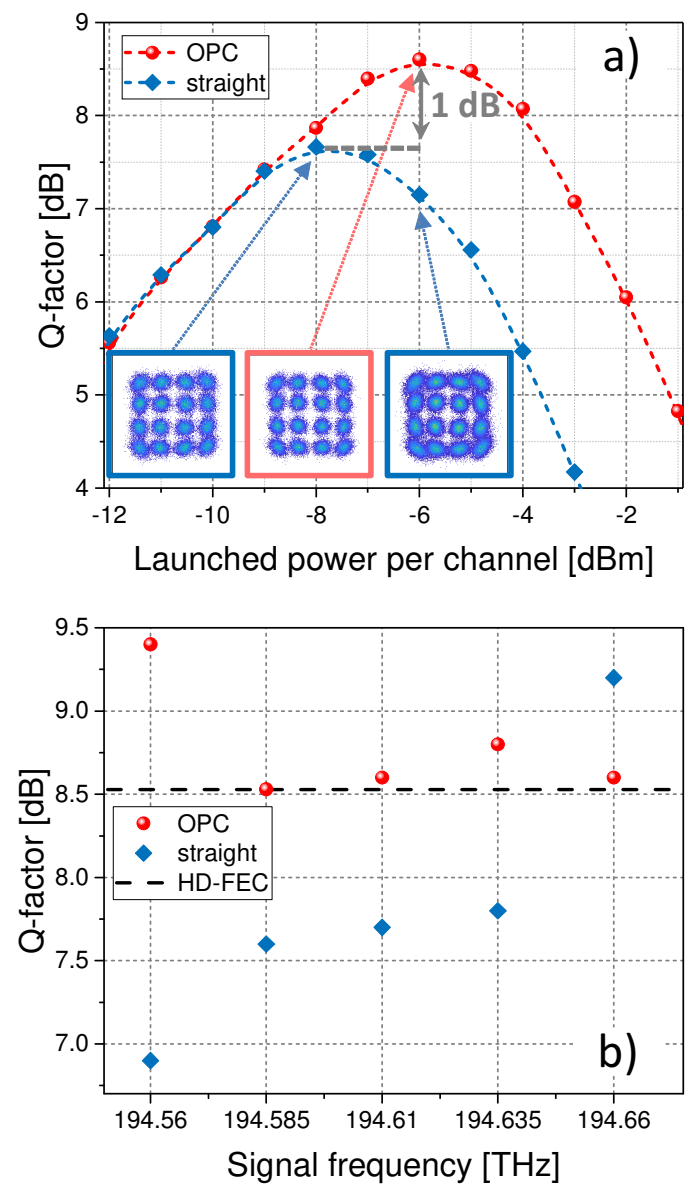

Fig. 7. Improvement provided by the OPC: (a) Q-factor for the central channel as a function of the input signal power per channel both in straight (w/o OPC) and OPC-based transmission. The inset constellation diagrams refer to the straight transmission at $-8 \mathrm{dBm}$ (left), the OPC-based transmission at $-6 \mathrm{dBm}$ (middle), and the straight at $-6 \mathrm{dBm}$ (right). (b) Q-factor for all the channels at optimum launch power with and without OPC.

OPC module based on a silicon nano-rib waveguide. The reduced free carrier effects, owing to the diode, enabled significant improvement in $\mathrm{CE}$, thus enabling the lowpenalty conversion, which is fundamental for including the 
OPC stage within a transmission scenario. By overcoming the limitations imposed by a conversion penalty [17, the nonlinearity compensation provided by the OPC leads to a 1-dB Q-factor improvement after 644-km of dispersion compensated transmission. This $\mathrm{Q}$ factor enhancement allowed for receiving all five tested WDM channels with a Qfactor above the HD-FEC threshold. This demonstration confirms the potential of silicon as nonlinear platform for all-optical signal processing within transmission systems, especially since the current limitations posed by the relatively narrow conversion bandwidth and high coupling loss can be expected to significantly improve with further design and fabrication optimization.

\section{Acknowledgment}

This work was supported by the DFG projects SFB 787, ZI 1283/-1 and PE319/36.1, the DNRF Center of Excellence, SPOC (ref. DNRF123), and DFF project NANO-SPECs (DFF-4005-00558B).

\section{References}

[1] A.D. Ellis, M.E. McCarthy, M.A.Z. Al Khateeb, M. Sorokina, and N.J. Doran, "Performance limits in optical communications due to fiber nonlinearity," Advances in Optics and Photonics 9(3), 429-503 (2017).

[2] J.C. Cartledge, F.P. Guiomar, F.R. Kschischang, G. Liga, and M.P. Yankov, "Digital Signal Processing for Fiber Nonlinearities," Optics Express, 25(3), 1916-1936 (2017).

[3] E. Ip and J.M. Kahn, "Compensation of dispersion and nonlinear impairments using digital backpropagation," Journal of Lightwave Technology 26(20), 34163425 (2008)

[4] D. Rafique and A.D. Ellis, "Impact of signal-ASE four-wave mixing on the effectiveness of digital back-propagation in 112 Gb/s PM-QPSK systems" Optics Express, 19(4), 34493454 (2011).

[5] G. Liga, T. Xu, A. Alvarado, R.I. Killey, and P. Bayvel, "On the performance of multichannel digital backpropagation in high-capacity long-haul optical transmission," Optics Express, 22(24), 3005330062 (2014).

[6] I. Sackey, et al., "Kerr nonlinearity mitigation: mid-link spectral inversion versus digital backpropagation in 5x28-GBd PDM 16QAM signal transmission," Journal of Lightwave Technology 33(9), 1821-1827 (2015).

[7] R. Dar and P. Winzer, "On the limits of digital backpropagation in fully loaded WDM systems," IEEE Photonics Technology Letters, 28(11), 12531256 (2016).

[8] N.K. Fontaine, et al., "Fiber nonlinearity comepnsation by digital backpropagation of an entire $1.2 \mathrm{~Tb} / \mathrm{s}$ superchannel using a full-field spectrally-sliced receiver," in Proc. ECOC 2013, paper Mo.3.D.5

[9] I. Sackey, et al., "Kerr nonlinearity mitigation in 528-GBd PDM 16-QAM signal transmission over a dispersion-uncompensated link with backward-pumped distributed Raman amplification," Optics Express 22(22), 2738127391 (2014).

[10] K. Solis-Trapala, et al., "Doubled transmission reach for DP64QAM signal over field-deployed legacy fiber systems enabled by MSSI," in Proc. ECOC 2015, paper Mo.3.6.2.

[11] A.D. Ellis, M.E. McCarthy, M.A.Z. Al-Khateeb, and S. Sygletos, "Capacity limits of systems employing multiple optical phase conjugators," Optics Express, 23(16), 2038120393 (2015).

[12] A.D. Ellis, et al., " $4 \mathrm{~Tb} / \mathrm{s}$ transmission reach enhancement using $10400 \mathrm{~Gb} / \mathrm{s}$ super-channels and polarization insensitive dual band optical phase conjugation," Journal of Lightwave Technology 34(8), 1717-1723 (2016).

[13] Y. Sun, et al., "Optical nonlinearity mitigation of 610GBd polarization-division multiplexing 16-QAM signals in a fieldinstalled transmission link," in Proc. OFC 2017, paper Th3J.2.

[14] I. Sackey, et al., "Waveband-shift-free optical phase conjugator for spectrally efficient fiber nonlinearity mitigation," Journal of Lightwave Technology 36(6), 1309-1317 (2018).
[15] A. Gajda, et al., "Silicon waveguide with lateral p-i-n Diode for nonlinearity compensation by On-Chip optical phase conjugation," in Proc. OFC 2018, paper W3E.4.

[16] T. Umeki, et al., "Simultaneous nonlinearity mitigation in 92 180-Gbit/s PDM-16QAM transmission over $3840 \mathrm{~km}$ using PPLN-based guard-band-less optical phase conjugation," Optics Express 24(15), 16945-16951 (2016).

[17] D. Vukovic, et al., "Multichannel nonlinear distortion compensation using optical phase conjugation in a silicon nanowire," Optics Express 23(3), 36403646 (2015).

[18] M.P. Yankov, F. Da Ros, E.P. da Silva, M. Galili, and L.K. Oxenløwe, "Experimental comparison of probabilistic shaping with online PMF optimization and mid-link OPC," in Proc. CLEO 2018, paper STu4C.2.

[19] C.R. Doerr, "Integrated Silicon Photonics," in Proc. ECOC 2017, paper W.1.C.1.

[20] D. Thomson, et al., "Roadmap on silicon photonics," Journal of Optics, 18(7), 073003 (2016).

[21] S.M. Hendrickson, A.C. Foster, R.M. Camacho, and B.D. Clader, "Integrated nonlinear photonics: emerging applications and ongoing challenges," Journal of the Optical Society of America B, 31(12) 3193-3203 (2014).

[22] K.-Y. Wang, and A.C. Foster, "GHz-rate optical parametric amplifier in hydrogenated amorphous silicon," Journal of Optics, 17(9), 094012 (2015).

[23] P.D. Girouard, L.H. Frandsen, M. Galili, and L.K. Oxenløwe, "A deuterium-passivated amorphous silicon platform for stable integrated nonlinear optics," in Proc. CLEO 2018, paper SW4I.2.

[24] C. Lacava et al., "Nonlinear silicon photonic signal processing devices for future optical networks," Applied Science, 7(1) 103 (2017).

[25] M.A. Ettabib et al., "FWM-based wavelength conversion of 40 Gbaud PSK signals in a silicon germanium waveguide," Optics Express, 21(14), 16683-16689 (2013).

[26] C. Lacava, et al., "Si-rich silicon nitride for nonlinear signal processing applications," Scientific Reports, 7(22), 2045-2322 (2017).

[27] F. Da Ros, et al., "Wavelength conversion of QAM signals in a low loss CMOS compatible spiral waveguide," APL Photonics, 2(4) 046105 (2017).

[28] A.C. Turner-Foster, et al., "Ultrashort free-carrier lifetime in low-loss silicon nanowaveguides," Optics Express, 18(4), 35823591 (2010).

[29] A. Gajda, L. Zimmermann, J. Bruns, B. Tillack and K. Petermann, "Design rules for p-i-n diode carriers sweeping in nano-rib waveguides on SOI ," Optics Express, 19(10), 99159922 (2011).

[30] A. Gajda, et al., "Highly efficient CW parametric conversion at $1550 \mathrm{~nm}$ in SOI waveguides by reverse biased p-i-n junction," Optics Expres 20(12), 13100-13107 (2012).

[31] I. Sackey, et al. "1.024 Tb/s wavelength conversion in a silicon waveguide with reverse-biased p-i-n junction," Optics Express 25(18), 21229-21240 (2017).

[32] F. Van Laere, et al., "Focusing Polarization Diversity Grating Couplers in Silicon-on-Insulator," Journal of Lightwave Technology 27(5), 612-618 (2009).

[33] F. Da Ros, et al., "Dual-polarization wavelength conversion of 16-QAM signals in a single silicon waveguide with a lateral p-i-n diode," Photonics Research 6(5) B23-B29 (2018).

[34] P.M. Kaminski, et al, "Signal-to-idler conversion penalty in AlGaAs-on-Insulator wavelength converter," in Proc. CLEO 2018, paper STu4C.6.

[35] Y. Sun, "Polarization effects in a recirculating loop system and emulation of a straigh-line system using a recirculating loop," Ph.D. dissertation, 2003.

[36] M.E. McCarthy, M.A.Z. Al-Kahteeb, F.M.Ferreira, and A.D. Ellis "PMD tolerant nonlinear compensation using in-line phase conjugation," Optics Express 24(4) 3385-3392 (2016). 